\title{
SHARP INTEGRAL INEQUALITIES BASED ON GENERAL EULER TWO-POINT FORMULAE
}

\author{
J. PEČARIĆ ${ }^{1}$, I. PERIĆ ${ }^{2}$ and A. VUKELIĆ ${ }^{2}$
}

(Received 9 April, 2003; revised 24 April, 2004)

\begin{abstract}
We consider a family of two-point quadrature formulae, using some Euler-type identities. A number of inequalities, for functions whose derivatives are either functions of bounded variation, Lipschitzian functions or $R$-integrable functions, are proved.
\end{abstract}

\section{Introduction}

In the recent paper [5] the following two identities, named the extended Euler formulae, have been proved. For $n \geq 1$ and every $x \in[0,1]$

and

$$
f(x)=\int_{0}^{1} f(t) d t+T_{n}(x)+R_{n}^{1}(x)
$$

$$
f(x)=\int_{0}^{1} f(t) d t+T_{n-1}(x)+R_{n}^{2}(x),
$$

where $T_{0}(x)=0$ and

$$
T_{m}(x)=\sum_{k=1}^{m} \frac{B_{k}(x)}{k !}\left[f^{(k-1)}(1)-f^{(k-1)}(0)\right],
$$

for $1 \leq m \leq n$, while

$$
\begin{aligned}
& R_{n}^{1}(x)=-\frac{1}{n !} \int_{0}^{1} B_{n}^{*}(x-t) d f^{(n-1)}(t), \\
& R_{n}^{2}(x)=-\frac{1}{n !} \int_{0}^{1}\left[B_{n}^{*}(x-t)-B_{n}(x)\right] d f^{(n-1)}(t) .
\end{aligned}
$$

\footnotetext{
'Faculty of Textile Technology, University of Zagreb, Pierottijeva 6, 10000 Zagreb, Croatia: e-mail: pecaric@mahazu.hazu.hr.

${ }^{2}$ Faculty of Food Technology and Biotechnology, Mathematics Department, University of Zagreb, Pierottijeva 6, 10000 Zagreb, Croatia; e-mail: iperic@pbf.hr and avukelic@pbf.hr.

(C) Australian Mathematical Society 2005, Serial-fee code 1446-1811/05
} 
Here, as in the rest of the paper, we write $\int_{0}^{1} g(t) d \varphi(t)$ to denote the Riemann-Stieltjes integral with respect to a function $\varphi:[0,1] \rightarrow \mathbb{R}$ of bounded variation, and $\int_{0}^{1} g(t) d t$ for the Riemann integral. The identities (1.1) and (1.2) extend the well-known formula for the expansion of a function in Bernoulli polynomials [15, page 17]. They hold for every function $f:[0,1] \rightarrow \mathbb{R}$ such that $f^{(n-1)}$ is a continuous function of bounded variation on $[0,1]$. The functions $B_{k}(t)$ are the Bernoulli polynomials, $B_{k}=B_{k}(0)$ are the Bernoulli numbers, and $B_{k}^{*}(t), k \geq 0$, are periodic functions of period 1 , related to the Bernoulli polynomials as

$$
B_{k}^{*}(t)=B_{k}(t), \quad 0 \leq t<1 \quad \text { and } \quad B_{k}^{*}(t+1)=B_{k}^{*}(t), \quad t \in \mathbb{R} .
$$

The Bernoulli polynomials $B_{k}(t), k \geq 0$, are uniquely determined by the following identities:

$$
\begin{array}{lll}
B_{k}^{\prime}(t)=k B_{k-1}(t), & k \geq 1 \\
B_{0}(t)=1, & B_{k}(t+1)-B_{k}(t)=k t^{k-1}, & k \geq 0 .
\end{array}
$$

For some further details on the Bernoulli polynomials and the Bernoulli numbers see for example [1] or [2]. We have that $B_{0}^{*}(t)=1$ and $B_{1}^{*}(t)$ is a discontinuous function with a jump of -1 at each integer. It follows that $B_{k}(1)=B_{k}(0)=B_{k}$ for $k \geq 2$, so that $B_{k}^{*}(t)$ are continuous functions for $k \geq 2$. We get

$$
B_{k}^{* \prime}(t)=k B_{k-1}^{*}(t), \quad k \geq 1,
$$

for every $t \in \mathbb{R}$ when $k \geq 3$, and for every $t \in \mathbb{R} \backslash \mathbb{Z}$ when $k=1,2$. In this paper we study, for each real number $x \in[0,1 / 2]$, the general two-point quadrature formula

$$
\int_{0}^{1} f(t) d t=\frac{1}{2}[f(x)+f(1-x)]+E(f ;, x),
$$

with $E(f ; x)$ being the remainder. This family of two-point quadrature formulae was considered by Guessab and Schmeisser in [14] and they established sharp estimates for the remainder under various regularity conditions. The aim of this paper is to establish a general two-point formula (1.5) using identities (1.1)-(1.2) and to give various error estimates for the quadrature rules based on such generalisations. In Section 2 we use the extended Euler formulae to obtain two new integral identities. We call them the general Euler two-point formulae. In Section 3, we prove a number of inequalities which give error estimates for the general Euler two-point formulae for functions whose derivatives are from the $L_{p}$-spaces, thus we extend the results from [14] and we generalise the results from papers $[6-8,16]$ and $[17]$. These inequalities are generally sharp (in the case $p=1$ the best possible). Special attention is devoted to the case where we have some boundary conditions and in some cases we compare our estimates with Fink's estimates $([13,14])$. 


\section{General Euler two-point formulae}

For $k \geq 1$ and fixed $x \in[0,1 / 2]$ define the functions $G_{k}^{x}(t)$ and $F_{k}^{x}(t)$ as

$$
G_{k}^{x}(t)=B_{k}^{*}(x-t)+B_{k}^{*}(1-x-t), \quad t \in \mathbb{R}
$$

and $F_{k}^{x}(t)=G_{k}^{x}(t)-\tilde{B}_{k}(x), t \in \mathbb{R}$, where

$$
\tilde{B}_{k}(x)=B_{k}(x)+B_{k}(1-x), \quad x \in[0,1 / 2], k \geq 1 .
$$

In particular, we get $\tilde{B}_{1}(x)=0, \tilde{B}_{2}(x)=2 x^{2}-2 x+1 / 3$ and $\tilde{B}_{3}(x)=0$. Also, for $k \geq 2$ we have $\tilde{B}_{k}(x)=G_{k}^{x}(0)$, that is, $F_{k}^{x}(t)=G_{k}^{x}(t)-G_{k}^{x}(0), k \geq 2$, and $F_{1}^{x}(t)=G_{1}^{x}(t), t \in \mathbb{R}$. Obviously, $G_{k}^{x}(t)$ and $F_{k}^{x}(t)$ are periodic functions of period 1 and continuous for $k \geq 2$.

Let $f:[0,1] \rightarrow \mathbb{R}$ be such that $f^{(n-1)}$ exists on $[0,1]$ for some $n \geq 1$. We introduce the following notation for each $x \in[0,1 / 2]$ :

$$
D(x)=[f(x)+f(1-x)] / 2 .
$$

Further, we define $\tilde{T}_{0}(x)=0$ and, for $1 \leq m \leq n, x \in[0,1 / 2]$,

$$
\tilde{T}_{m}(x)=\left[T_{m}(x)+T_{m}(1-x)\right] / 2,
$$

where $T_{m}(x)$ is given by (1.3). It is easy to see that

$$
\tilde{T}_{m}(x)=\frac{1}{2} \sum_{k=1}^{m} \frac{\tilde{B}_{k}(x)}{k !}\left[f^{(k-1)}(1)-f^{(k-1)}(0)\right] .
$$

In the next theorem we establish two formulae which play a key role in this paper. We call them the general Euler two-point formulae.

THEOREM 2.1. Let $f:[0,1] \rightarrow \mathbb{R}$ be such that $f^{(n-1)}$ is a continuous function of bounded variation on $[0,1]$, for some $n \geq 1$. Then for each $x \in[0,1 / 2]$

$$
\int_{0}^{1} f(t) d t=D(x)-\tilde{T}_{n}(x)+\tilde{R}_{n}^{\prime}(f)
$$

and

$$
\int_{0}^{1} f(t) d t=D(x)-\tilde{T}_{n-1}(x)+\tilde{R}_{n}^{2}(f)
$$

where

$$
\tilde{R}_{n}^{1}(f)=\frac{1}{2(n !)} \int_{0}^{1} G_{n}^{x}(t) d f^{(n-1)}(t), \quad \tilde{R}_{n}^{2}(f)=\frac{1}{2(n !)} \int_{0}^{1} F_{n}^{x}(t) d f^{(n-1)}(t) .
$$


Proof. Put $x \equiv x$ and $x \equiv 1-x$ in formula (1.1) to get two new formulae. Then multiply these new formulae by $1 / 2$ and add. The result is formula (2.2). Formula (2.3) is obtained from (1.2) by the same procedure.

REMARK 1. If in Theorem 2.1 we choose $x=0,1 / 2,1 / 3,1 / 4$ we get the Euler trapezoid [6], the Euler midpoint [8], the Euler two-point Newton-Cotes [17] and the Euler two-point Maclaurin formulae respectively.

By direct calculations for each $x \in[0,1 / 2]$, we get

$$
\begin{aligned}
F_{1}^{x}(t)=G_{1}^{x}(t) & = \begin{cases}-2 t, & 0 \leq t \leq x ; \\
-2 t+1, & x<t \leq 1-x ; \\
-2 t+2, & 1-x<t \leq 1,\end{cases} \\
G_{2}^{x}(t) & = \begin{cases}2 t^{2}+2 x^{2}-2 x+1 / 3, & 0 \leq t \leq x ; \\
2 t^{2}-2 t+2 x^{2}+1 / 3, & x<t \leq 1-x ; \\
2 t^{2}-4 t+2 x^{2}-2 x+7 / 3, & 1-x<t \leq 1,\end{cases} \\
F_{2}^{x}(t) & = \begin{cases}2 t^{2}, & 0 \leq t \leq x ; \\
2 t^{2}-2 t+2 x, & x<t \leq 1-x ; \\
2 t^{2}-4 t+2, & 1-x<t \leq 1\end{cases}
\end{aligned}
$$

and

$$
F_{3}^{x}(t)=G_{3}^{x}(t)= \begin{cases}-2 t^{3}+\left(-6 x^{2}+6 x-1\right) t, & 0 \leq t \leq x \\ -2 t^{3}+3 t^{2}+\left(-6 x^{2}-1\right) t+3 x^{2}, & x<t \leq 1-x \\ -2 t^{3}+6 t^{2}+\left(-6 x^{2}+6 x-7\right) t & \\ +6 x^{2}-6 x+3, & 1-x<t \leq 1\end{cases}
$$

We now will prove some properties of the functions $G_{k}^{x}(t)$ and $F_{k}^{x}(t)$ defined above. The Bernoulli polynomials are symmetric with respect to $1 / 2$, (see [1]), that is,

$$
B_{k}(1-x)=(-1)^{k} B_{k}(x), \quad k \geq 1 .
$$

Also, we have $B_{k}(1)=B_{k}(0)=B_{k}, k \geq 2, B_{1}(1)=-B_{1}(0)=1 / 2$ and $B_{2 j-1}=0$, $j \geq 2$. Therefore we get $\tilde{B}_{2 j-1}(x)=0, j \geq 1$ and $\tilde{B}_{2 j}(x)=2 B_{2 j}(x), x \in[0,1 / 2]$. Now, we have $F_{2 j-1}^{x}(t)=G_{2 j-1}^{x}(t), j \geq 1$, and

$$
F_{2 j}^{x}(t)=G_{2 j}^{x}(t)-\tilde{B}_{2 j}(x)=G_{2 j}^{x}(t)-2 B_{2 j}(x), \quad x \in[0,1 / 2], j \geq 1 .
$$

Further, the points 0 and 1 are the zeros of $F_{k}^{x}(t)=G_{k}^{x}(t)-G_{k}^{x}(0), k \geq 2$, that is, $F_{k}^{x}(0)=F_{k}^{x}(1)=0, k \geq 1$. As we shall see below, 0 and 1 are the only zeros of $F_{2 j}^{x}(t)$ for $j \geq 2$ and $x \in[0,1 / 2-1 / 2 \sqrt{3}) \cup(1 / 2 \sqrt{3}, 1 / 2]$. Next, setting $t=1 / 2$ 
in (2.8) we get $B_{k}(1 / 2)=(-1)^{k} B_{k}(1 / 2), k \geq 1$, which implies that $B_{2 j-1}(1 / 2)=0$, $j \geq 1$. Using the above formulae, we get $F_{2 j-1}^{x}(1 / 2)=G_{2 j-1}^{x}(1 / 2)=0, j \geq 1$. We shall see that $0,1 / 2$ and 1 are the only zeros of $F_{2 j-1}^{x}(t)=G_{2 j-1}^{x}(t)$, for $j \geq 2$ and $x \in[0,1 / 2-1 / 2 \sqrt{3}) \cup(1 / 2 \sqrt{3}, 1 / 2]$. Also, note that for $x \in[0,1 / 2], j \geq 1$, $G_{2 j}^{x}(1 / 2)=2 B_{2 j}(1 / 2-x)$ and

$$
F_{2 j}^{x}(1 / 2)=G_{2 j}^{x}(1 / 2)-\tilde{B}_{2 j}(x)=2 B_{2 j}(1 / 2-x)-2 B_{2 j}(x) .
$$

LEMMA 2.2. For $k \geq 2$ we have $G_{k}^{x}(1-t)=(-1)^{k} G_{k}^{x}(t), 0 \leq t \leq 1$ and $F_{k}^{x}(1-t)=(-1)^{k} F_{k}^{x}(t), 0 \leq t \leq 1$.

Proof. As the functions $B_{k}^{*}(t)$ are periodic with period 1 and continuous for $k \geq 2$, similar to $[6,8,16]$ and $[17]$ we get these two identities.

Note that the identities established in Lemma 2.2 are valid for $k=1$, too, except at the points $x$ and $1-x$ of discontinuity of $F_{1}^{x}(t)=G_{1}^{x}(t)$.

LEMMA 2.3. For $k \geq 2$ and $x \in[0,1 / 2-1 / 2 \sqrt{3}) \cup(1 / 2 \sqrt{3}, 1 / 2]$ the function $G_{2 k-1}^{x}(t)$ has no zeros in the interval $(0,1 / 2)$. For $0<t<1 / 2$ the sign of this function is determined by

$$
\begin{aligned}
& (-1)^{k-1} G_{2 k-1}^{x}(t)>0, \quad x \in[0,1 / 2-1 / 2 \sqrt{3}) \text { and } \\
& (-1)^{k} G_{2 k-1}^{x}(t)>0, \quad x \in(1 / 2 \sqrt{3}, 1 / 2] \text {. }
\end{aligned}
$$

Proof. For $k=2, G_{3}^{x}(t)$ is given by (2.7) and it is easy to see that for $0<t<1 / 2$, $G_{3}^{x}(t)<0, x \in[0,1 / 2-1 / 2 \sqrt{3})$ and $G_{3}^{x}(t)>0, x \in(1 / 2 \sqrt{3}, 1 / 2]$. Thus our assertion is true for $k=2$. Now, using a simple induction similar to that in $[6,8,16]$ and [17] we prove that $G_{2 k-1}^{x}(t)$ cannot have a zero inside the interval $(0,1 / 2)$. To determine the sign of $G_{2 k-1}^{x}(t)$, note that $G_{2 k-1}^{x}(x)=B_{2 k-1}(1-2 x)$. We have [1, 23.1.14], $(-1)^{k} B_{2 k-1}(t)>0,0<t<1 / 2$, which implies for $x \in[0,1 / 2-1 / 2 \sqrt{3})$

$$
(-1)^{k-1} G_{2 k-1}^{x}(x)=(-1)^{k-1} B_{2 k-1}(1-2 x)=(-1)^{k} B_{2 k-1}(2 x)>0
$$

and for $x \in(1 / 2 \sqrt{3}, 1 / 2]$

$$
(-1)^{k} G_{2 k-1}^{x}(x)=(-1)^{k} B_{2 k-1}(1-2 x)>0,
$$

which completes the proof.

COROLlaRY 2.4. For $k \geq 2$ and $x \in[0,1 / 2-1 / 2 \sqrt{3})$ the functions $(-1)^{k} F_{2 k}^{x}(t)$ and $(-1)^{k} G_{2 k}^{x}(t)$ are strictly increasing on the interval $(0,1 / 2)$ and strictly decreasing on the interval $(1 / 2,1)$. Also, for $x \in(1 / 2 \sqrt{3}, 1 / 2]$ the functions $(-1)^{k-1} F_{2 k}^{x}(t)$ and 
$(-1)^{k-1} G_{2 k}^{x}(t)$ are strictly increasing on the interval $(0,1 / 2)$, and strictly decreasing on the interval $(1 / 2,1)$. Further, for $k \geq 2$, we have

and

$$
\max _{t \in[0,1]}\left|F_{2 k}^{x}(t)\right|=2\left|B_{2 k}(1 / 2-x)-B_{2 k}(x)\right|
$$

$$
\max _{t \in[0,1]}\left|G_{2 k}^{x}(t)\right|=2 \max \left\{\left|B_{2 k}(x)\right|,\left|B_{2 k}(1 / 2-x)\right|\right\}
$$

PROOF. Using (1.4) we get $\left[(-1)^{k} F_{2 k}^{x}(t)\right]^{\prime}=\left[(-1)^{k} G_{2 k}^{x}(t)\right]^{\prime}=2 k(-1)^{k-1} G_{2 k-1}^{x}(t)$ and $(-1)^{k-1} G_{2 k-1}^{x}(t)>0$ for $0<t<1 / 2$ and $x \in[0,1 / 2-1 / 2 \sqrt{3})$, by Lemma 2.3 . Thus $(-1)^{k} F_{2 k}^{x}(t)$ and $(-1)^{k} G_{2 k}^{x}(t)$ are strictly increasing on the interval $(0,1 / 2)$. Also, by Lemma 2.2, we have $F_{2 k}^{x}(1-t)=F_{2 k}^{x}(t), 0 \leq t \leq 1$ and $G_{2 k}^{x}(1-t)=G_{2 k}^{x}(t)$, $0 \leq t \leq 1$, which implies that $(-1)^{k} F_{2 k}^{x}(t)$ and $(-1)^{k} G_{2 k}^{x}(t)$ are strictly decreasing on the interval $(1 / 2,1)$. The proof of the second statement is similar. Further, $F_{2 k}^{x}(0)=F_{2 k}^{x}(1)=0$, which implies that $\left|F_{2 k}^{x}(t)\right|$ achieves its maximum at $t=1 / 2$, that is, $\max _{t \in[0,1]}\left|F_{2 k}^{x}(t)\right|=\left|F_{2 k}^{x}(1 / 2)\right|=2\left|B_{2 k}(1 / 2-x)-B_{2 k}(x)\right|$. Also

$$
\max _{i \in[0,1]}\left|G_{2 k}^{x}(t)\right|=\max \left\{\left|G_{2 k}^{x}(0)\right|,\left|G_{2 k}^{x}(1 / 2)\right|\right\}=2 \max \left\{\left|B_{2 k}(x)\right|,\left|B_{2 k}(1 / 2-x)\right|\right\},
$$

which completes the proof.

COROLLARY 2.5. For $k \geq 2$, and $x \in[0,1 / 2-1 / 2 \sqrt{3}) \cup(1 / 2 \sqrt{3}, 1 / 2]$ we have Also, we have

$$
\int_{0}^{1}\left|F_{2 k-1}^{x}(t)\right| d t=\int_{0}^{1}\left|G_{2 k-1}^{x}(t)\right| d t=\frac{2}{k}\left|B_{2 k}(1 / 2-x)-B_{2 k}(x)\right| .
$$

$$
\begin{aligned}
& \int_{0}^{1}\left|F_{2 k}^{x}(t)\right| d t=\left|\tilde{B}_{2 k}(x)\right|=2\left|B_{2 k}(x)\right| \text { and } \\
& \int_{0}^{1}\left|G_{2 k}^{x}(t)\right| d t \leq 2\left|\tilde{B}_{2 k}(x)\right|=4\left|B_{2 k}(x)\right| .
\end{aligned}
$$

ProOF. Using Lemmas 2.2-2.3 we get

$$
\begin{aligned}
\int_{0}^{1}\left|G_{2 k-1}^{x}(t)\right| d t & =2\left|\int_{0}^{1 / 2} G_{2 k-1}^{x}(t) d t\right|=2\left|-\frac{1}{2 k} G_{2 k}^{x}(t)\right|_{0}^{1 / 2} \mid \\
& =\frac{1}{k}\left|G_{2 k}^{x}(1 / 2)-G_{2 k}^{x}(0)\right|=\frac{2}{k}\left|B_{2 k}(1 / 2-x)-B_{2 k}(x)\right|,
\end{aligned}
$$

which proves the first assertion. By Corollary 2.4 and because $F_{2 k}^{x}(0)=F_{2 k}^{x}(1)=0$, 
$F_{2 k}^{x}(t)$ does not change its sign on the interval $(0,1)$. Therefore using (2.9) we get

$$
\begin{aligned}
\int_{0}^{1}\left|F_{2 k}^{x}(t)\right| d t & =\left|\int_{0}^{1} F_{2 k}^{x}(t) d t\right|=\left|\int_{0}^{1}\left[G_{2 k}^{x}(t)-\tilde{B}_{2 k}(x)\right] d t\right| \\
& =\left|-\frac{1}{2 k+1} G_{2 k+1}^{x}(t)\right|_{0}^{1}-\tilde{B}_{2 k}(x)|=| \tilde{B}_{2 k}(x) \mid,
\end{aligned}
$$

which proves the second assertion. Finally, we use (2.9) again and the triangle inequality to obtain the third formula.

\section{Inequalities related to the general Euler two-point formulae}

In this section we use formulae established in Theorem 2.1 to prove a number of inequalities using $L_{p}$ norms for $1 \leq p \leq \infty$. These inequalities are generally sharp (in the case $p=1$ the best possible). Special attention is devoted to the case where we have some boundary conditions and in some cases we compare our constants with the Fink constants $([13,14])$.

THEOREM 3.1. Assume $(p, q)$ is a pair of conjugate exponents, $1 \leq p, q \leq \infty$. Let $\left|f^{(n)}\right|^{p}:[0,1] \rightarrow \mathbb{R}$ be an $R$-integrable function for some $n \geq 1$. Then for every $x \in[0,1 / 2]$, we have

$$
\begin{aligned}
\left|\int_{0}^{1} f(t) d t-D(x)+\tilde{T}_{n-1}(x)\right| & \leq K(n, p, x) \cdot\left\|f^{(n)}\right\|_{p} \quad \text { and } \\
\left|\int_{0}^{1} f(t) d t-D(x)+\tilde{T}_{n}(x)\right| & \leq K^{*}(n, p, x) \cdot\left\|f^{(n)}\right\|_{p},
\end{aligned}
$$

where

$$
\begin{aligned}
& K(n, p, x)=\frac{1}{2(n !)}\left[\int_{0}^{1}\left|F_{n}^{x}(t)\right|^{q} d t\right]^{1 / q}, \\
& K^{*}(n, p, x)=\frac{1}{2(n !)}\left[\int_{0}^{1}\left|G_{n}^{x}(t)\right|^{q} d t\right]^{1 / q} .
\end{aligned}
$$

The constants $K(n, p, x)$ and $K^{*}(n, p, x)$ are sharp for $1<p \leq \infty$ and the best possible for $p=1$.

Proof. Applying the Hölder inequality we have

$$
\begin{aligned}
\left|\frac{1}{2(n !)} \int_{0}^{1} F_{n}^{x}(t) f^{(n)}(t) d t\right| & \leq \frac{1}{2(n !)}\left[\int_{0}^{1}\left|F_{n}^{x}(t)\right|^{q} d t\right]^{1 / q}\left\|f^{(n)}\right\|_{p} \\
& =K(n, p, x)\left\|f^{(n)}\right\|_{p} .
\end{aligned}
$$


Using the above inequality, from (2.3) we get estimate (3.1). In the same manner, from (2.2) we get estimate (3.2). Now, we consider the optimality of $K(n, p, x)$. We shall find a function $f$ such that

$$
\left|\int_{0}^{1} F_{n}^{x}(t) f^{(n)} d t\right|=\left(\int_{0}^{1}\left|F_{n}^{x}(t)\right|^{q} d t\right)^{1 / q}\left(\int_{0}^{1}\left|f^{(n)}(t)\right|^{p} d t\right)^{1 / p} .
$$

For $1<p<\infty$ take $f$ to be such that

$$
f^{(n)}(t)=\operatorname{sgn} F_{n}^{x}(t)\left|F_{n}^{x}(t)\right|^{1 /(p-1)},
$$

where for $p=\infty$ we put $f^{(n)}(t)=\operatorname{sgn} F_{n}^{x}(t)$. For constant $K^{*}(n, p, x)$ the proof of sharpness is analogous. For $p=1$ we shall prove that

$$
\left|\int_{0}^{1} F_{n}^{x}(t) f^{(n)}(t) d t\right| \leq \max _{t \in[0,1]}\left|F_{n}^{x}(t)\right| \int_{0}^{1}\left|f^{(n)}(t)\right| d t
$$

is the best possible inequality. Suppose that $\left|F_{n}^{x}(t)\right|$ attains its maximum at $t_{0} \in(0,1)$. First, we assume that $F_{n}^{x}\left(t_{0}\right)>0$. For $\varepsilon$ small enough define $f_{\varepsilon}^{(n-1)}(t)$ by

$$
f_{\varepsilon}^{(n-1)}(t)= \begin{cases}0, & t \leq t_{0} \\ \left(t-t_{0}\right) / \varepsilon, & t \in\left[t_{0}, t_{0}+\varepsilon\right] \\ 1, & t \geq t_{0}+\varepsilon\end{cases}
$$

Then, for $\varepsilon$ small enough,

$$
\left|\int_{0}^{1} F_{n}^{x}(t) f_{\varepsilon}^{(n)}(t) d t\right|=\left|\int_{t_{0}}^{\omega_{0}+\varepsilon} F_{n}^{x}(t) \frac{1}{\varepsilon} d t\right|=\frac{1}{\varepsilon} \int_{t_{0}}^{t_{0}+\varepsilon} F_{n}^{x}(t) d t .
$$

Now, from inequality (3.4) we have

$$
\frac{1}{\varepsilon} \int_{t_{0}}^{t_{0}+\varepsilon} F_{n}^{x}(t) d t \leq F_{n}^{x}\left(t_{0}\right) \int_{t_{0}}^{t_{0}+\varepsilon} \frac{1}{\varepsilon} d t=F_{n}^{x}\left(t_{0}\right)
$$

Since

$$
\lim _{\varepsilon \rightarrow 0} \frac{1}{\varepsilon} \int_{t_{0}}^{t_{0}+\varepsilon} F_{n}^{x}(t) d t=F_{n}^{x}\left(t_{0}\right)
$$

the statement follows. If $F_{n}^{x}\left(t_{0}\right)<0$, then we take

$$
f_{\varepsilon}^{(n-1)}(t)= \begin{cases}1, & t \leq t_{0} \\ -\left(t-t_{0}-\varepsilon\right) / \varepsilon, & t \in\left[t_{0}, t_{0}+\varepsilon\right] ; \\ 0, & t \geq t_{0}+\varepsilon\end{cases}
$$

and the rest of the proof is the same as above. Proof of the best possibility of the second inequality is similar. 
REMARK 2. Basically we have three types of estimates:

$$
\begin{aligned}
& \left|\int_{0}^{1} f(t) d t-D(x)+\tilde{T}_{2 k}(x)\right| \\
& \quad \leq \frac{1}{2(2 k) !}\left(\int_{0}^{1}\left|G_{2 k}^{x}(t)\right|^{q} d t\right)^{1 / q}\left(\int_{0}^{1}\left|f^{(2 k)}(t)\right|^{p} d t\right)^{1 / p}, \\
& \left|\int_{0}^{1} f(t) d t-D(x)+\tilde{T}_{2 k}(x)\right| \\
& \quad \leq \frac{1}{2(2 k+1) !}\left(\int_{0}^{1}\left|G_{2 k+1}^{x}(t)\right|^{q} d t\right)^{1 / q}\left(\int_{0}^{1}\left|f^{(2 k+1)}(t)\right|^{p} d t\right)^{1 / p}
\end{aligned}
$$

and

$$
\begin{aligned}
& \left|\int_{0}^{1} f(t) d t-D(x)+\tilde{T}_{2 k}(x)\right| \\
& \quad \leq \frac{1}{2(2 k+2) !}\left(\int_{0}^{1}\left|F_{2 k+2}^{x}(t)\right|^{q} d t\right)^{1 / q}\left(\int_{0}^{1}\left|f^{(2 k+2)}(t)\right|^{p} d t\right)^{1 / p} .
\end{aligned}
$$

In the following theorem we are interested in the sharpness of the above estimates in the presence of boundary conditions.

THEOREM 3.2. Assume that $(p, q)$ is a pair of conjugate exponents, $1 \leq p, q \leq \infty$ and $k \in \mathbb{N}$. Let $f:[0,1] \rightarrow \mathbb{R}$ be a function such that we have boundary conditions $f^{(2 i-1)}(0)=f^{(2 i-1)}(1)$ for $i=1, \ldots, k$. Then for every $x \in[0,1 / 2]$ and $\left|f^{(2 k)}\right|^{p}$ $R$-integrable, we have

$$
\left|\int_{0}^{1} f(t) d t-D(x)\right| \leq \frac{1}{2(2 k) !}\left(\int_{0}^{1}\left|G_{2 k}^{x}(t)\right|^{q} d t\right)^{1 / q}\left(\int_{0}^{1}\left|f^{(2 k)}(t)\right|^{p} d t\right)^{1 / p} .
$$

For $\left[\left.f^{(2 k+1)}\right|^{p} R\right.$-integrable we have

$$
\left|\int_{0}^{1} f(t) d t-D(x)\right| \leq \frac{1}{2(2 k+1) !}\left(\int_{0}^{1}\left|G_{2 k+1}^{x}(t)\right|^{q} d t\right)^{1 / q}\left(\int_{0}^{1}\left|f^{(2 k+1)}(t)\right|^{p} d t\right)^{1 / p}
$$

and for $\left|f^{(2 k+2)} t\right|^{p} R$-integrable we have

$$
\left|\int_{0}^{1} f(t) d t-D(x)\right| \leq \frac{1}{2(2 k+2) !}\left(\int_{0}^{1}\left|F_{2 k+2}^{x}(t)\right|^{q} d t\right)^{1 / q}\left(\int_{0}^{1}\left|f^{(2 k+2)}(t)\right|^{p} d t\right)^{1 / p} .
$$

Inequality (3.5) is sharp for $p=2$ and inequalities (3.6) and (3.7) are sharp for $1<p \leq \infty$ and best possible for $p=1$. 
PROOF. Inequality (3.5) is sharp since a function $f$ for which we have equality in (3.2) in the case $p=2, n=2 k$ is defined by $f^{(2 k)}(t)=G_{2 k}^{x}(t)$, so we can choose $f$ such that

$$
\begin{aligned}
& f^{(2 k-1)}(t)=-\frac{1}{2 k+1} G_{2 k+1}^{x}(t), \\
& f^{(2 k-3)}(t)=-\frac{1}{(2 k+1)(2 k+2)(2 k+3)} G_{2 k+3}^{x}(t)
\end{aligned}
$$

and generally

$$
f^{(2 i-1)}(t)=-\frac{G_{4 k-2 i+1}^{x}(t)}{(2 k+1)(2 k+2) \cdots(4 k-2 i+1)}, \quad i=1, \ldots, k,
$$

which gives $f^{(2 i-1)}(0)=f^{(2 i-1)}(1)=0, i=1, \ldots, k$. In relation to the sharpness or the best possibility of inequality (3.6), notice first that approximation $\int_{0}^{1} f(t) d t \approx$ $D(x)-\tilde{T}_{2 k}(x)$ is exact of order $2 k+1$. Take any function $f$ which is optimal for inequality (3.1) in the case $n=2 k+1,1 \leq p \leq \infty$. Set

$$
g(t)=f(t)+\sum_{i=1}^{2 k} a_{i} t^{i}=f(t)+a_{2 k} t^{2 k}+a_{2 k-1} t^{2 k-1}+\cdots+a_{2} t^{2}+a_{1} t .
$$

We have $g^{(2 k-1)}(t)=f^{(2 k-1)}(t)+(2 k) ! a_{2 k} t+(2 k-1) ! a_{2 k-1}$ so

$$
\begin{aligned}
& 0=g^{(2 k-1)}(0)=f^{(2 k-1)}(0)+(2 k-1) ! a_{2 k-1}, \\
& 0=g^{(2 k-1)}(1)=f^{(2 k-1)}(1)+(2 k) ! a_{2 k}+(2 k-1) ! a_{2 k-1},
\end{aligned}
$$

which gives $a_{2 k}, a_{2 k-1}$. Using $g^{(2 k-3)}$ and conditions $g^{(2 k-3)}(0)=0=g^{(2 k-3)}(1)$ we analogously obtain $a_{2 k-2}, a_{2 k-3}$ and so on. So the function $g$ is also optimal for (3.1) and satisfies the boundary conditions $g^{(2 i-1)}(1)=g^{(2 i-1)}(0), i=1, \ldots, k$. Inequality (3.7) can be treated in the same way.

In the following we calculate the optimal constants in the cases $p=1, p=\infty$ and $p=2$.

COROLLARY 3.3. Let $f:[0,1] \rightarrow \mathbb{B}$ be given. If $f$ is L-Lipschitzian on $[0,1]$, then for each $x \in[0,1 / 2]$

$$
\left|\int_{0}^{1} f(t) d t-D(x)\right| \leq \frac{4 x^{2}+(1-2 x)^{2}}{4} L
$$

Proof. Using (2.4) for each $x \in[0,1 / 2]$ and applying (3.1) with $n=1$ and $p=\infty$ we get the above inequality. 
REMARK 3. The inequality (3.8) has been proved by Guessab and Schmeisser on the interval $[a, b]$ in [14] (see also [10]). They also proved that this inequality is sharp for each admissible $x$. Equality is attained exactly in the case of equality in Theorem 3.1 where we put $f^{\prime}(t)=\operatorname{sgn} F_{1}^{x}(t)$.

COROLlaRY 3.4. Let $f:[0,1] \rightarrow \mathbb{R}$ be given. If $f^{\prime}$ is L-Lipschitzian on $[0,1]$, then for each $x \in[0,1 / 4]$

$$
\left|\int_{0}^{1} f(t) d t-D(x)\right| \leq\left[-\frac{1}{2}\left(x^{2}-x+\frac{1}{6}\right)+\frac{1}{6}(1-4 x)^{3 / 2}\right] L
$$

and for each $x \in[1 / 4,1 / 2]$

$$
\left|\int_{0}^{1} f(t) d t-D(x)\right| \leq-\frac{1}{2}\left(x^{2}-x+\frac{1}{6}\right) L .
$$

Proof. Using (2.6) for each $x \in[0,1 / 4]$ and applying (3.1) with $n=2$ and $p=\infty$ we get the above inequalities.

REMARK 4. The inequalities (3.9) and (3.10) have been proved by Guessab and Schmeisser on the interval $[a, b]$ in [14]. They also proved that these inequalities are sharp for each admissible $x$.

COROLlaRY 3.5. Let $f:[0,1] \rightarrow \mathbb{R}$ be given. If $f^{\prime}$ is L-Lipschitzian on $[0,1]$, then for each $x \in[0,1 / 2-1 / 2 \sqrt{3}]$

$$
\left|\int_{0}^{1} f(t) d t-D(x)+\frac{B_{2}(x)}{2}\left[f^{\prime}(1)-f^{\prime}(0)\right]\right| \leq \frac{1}{18 \sqrt{3}}\left(1-12 x^{2}\right)^{3 / 2} L
$$

for each $x \in[1 / 2-1 / 2 \sqrt{3}, 1 / 2 \sqrt{3}]$

$$
\begin{aligned}
& \left|\int_{0}^{1} f(t) d t-D(x)+\frac{B_{2}(x)}{2}\left[f^{\prime}(1)-f^{\prime}(0)\right]\right| \\
& \quad \leq\left[\frac{4}{3}\left(-x^{2}+x-\frac{1}{6}\right)^{3 / 2}+\frac{1}{18 \sqrt{3}}\left(1-12 x^{2}\right)^{3 / 2}\right] L
\end{aligned}
$$

and for each $x \in[1 / 2 \sqrt{3}, 1 / 2]$

$$
\left|\int_{0}^{1} f(t) d t-D(x)+\frac{B_{2}(x)}{2}\left[f^{\prime}(1)-f^{\prime}(0)\right]\right| \leq \frac{4}{3}\left(-x^{2}+x-\frac{1}{6}\right)^{3 / 2} L .
$$


PROof. Using (2.5) for each $x \in[0,1 / 2-1 / 2 \sqrt{3}]$ we get

$$
\begin{aligned}
\int_{0}^{1}\left|G_{2}^{x}(t)\right| d t & =2 \int_{0}^{1 / 2}\left|G_{2}^{x}(t)\right| d t \\
& =2\left[-\int_{0}^{1 / 2} G_{2}^{x}(t) d t+2 \int_{0}^{1 / 2-\sqrt{1-12 x^{2}} / 2 \sqrt{3}} G_{2}^{x}(t) d t\right] \\
& =4 \int_{0}^{1 / 2-\sqrt{1-12 x^{2}} / 2 \sqrt{3}} G_{2}^{x}(t) d t \\
& =4\left(-\left.\frac{1}{3} G_{3}^{x}(t)\right|_{0} ^{1 / 2-\sqrt{1-12 x^{2}} / 2 \sqrt{3}}\right)=-\frac{4}{3} G_{3}^{x}\left(\frac{1}{2}-\frac{\sqrt{1-12 x^{2}}}{2 \sqrt{3}}\right)
\end{aligned}
$$

for each $x \in[1 / 2-1 / 2 \sqrt{3}, 1 / 2 \sqrt{3}]$

$$
\begin{aligned}
\int_{0}^{1}\left|G_{2}^{x}(t)\right| d t & =4 \int_{\sqrt{-x^{2}+x+1 / 6}}^{1 / 2-\sqrt{1-12 x^{2}} / 2 \sqrt{3}} G_{2}^{x}(t) d t \\
& =\frac{4}{3}\left[G_{3}^{x}\left(\sqrt{-x^{2}+x-\frac{1}{6}}\right)-G_{3}^{x}\left(\frac{1}{2}-\frac{\sqrt{1-12 x^{2}}}{2 \sqrt{3}}\right)\right]
\end{aligned}
$$

and for each $x \in[1 / 2 \sqrt{3}, 1 / 2]$ we get

$$
\int_{0}^{1}\left|G_{2}^{x}(t)\right| d t=-4 \int_{0}^{\sqrt{-x^{2}+x-1 / 6}} G_{2}^{x}(t) d t=\frac{4}{3} G_{3}^{x}\left(\sqrt{-x^{2}+x-1 / 6}\right) .
$$

Therefore, applying (3.2) with $n=2$ and $p=\infty$, we get the above inequalities.

REMARK 5. In Theorem 3.2 it was proved that (3.5) is sharp just for $p=2$. We mention here that comparing the sharp constant from Guessab and Schmeisser in [14] in the case $p=\infty$ and our constant, we conclude that inequality (3.5) is not generally sharp. Namely, our constant for boundary conditions $f^{\prime}(1)=f^{\prime}(0)$, $n=2$ and $x=0$ is $1 /(18 \sqrt{3}$ ), while they have $1 / 32$ (note that the sharpness of (3.5) under conditions $f^{\prime}(1)=f^{\prime}(0)$ implies the sharpness of the same inequality under conditions $\left.f^{\prime}(1)=f^{\prime}(0)=0\right)$.

COROLlaRY 3.6. Let $f:[0,1] \rightarrow \mathbb{R}$ be given. If $f^{\prime \prime}$ is L-Lipschitzian on $[0,1]$, then for each $x \in[0,1 / 2-1 / 2 \sqrt{3}]$

$$
\left|\int_{0}^{1} f(t) d t-D(x)+\frac{B_{2}(x)}{2}\left[f^{\prime}(1)-f^{\prime}(0)\right]\right| \leq\left(\frac{x^{3}}{6}-\frac{x^{2}}{8}+\frac{1}{192}\right) L,
$$


for each $x \in[1 / 2-1 / 2 \sqrt{3}, 1 / 4]$

$$
\begin{aligned}
& \left|\int_{0}^{1} f(t) d t-D(x)+\frac{B_{2}(x)}{2}\left[f^{\prime}(1)-f^{\prime}(0)\right]\right| \\
& \quad \leq\left[\frac{x^{3}}{6}-\frac{x^{2}}{8}+\frac{1}{192}+\frac{1}{6}\left(-3 x^{2}+3 x-\frac{1}{2}\right)^{2}\right] L,
\end{aligned}
$$

for each $x \in[1 / 4,1 / 2 \sqrt{3}]$

$$
\begin{aligned}
& \left|\int_{0}^{1} f(t) d t-D(x)+\frac{B_{2}(x)}{2}\left[f^{\prime}(1)-f^{\prime}(0)\right]\right| \\
& \quad \leq\left[-\frac{x^{3}}{6}+\frac{x^{2}}{8}-\frac{1}{192}+\frac{1}{96}\left(1-12 x^{2}\right)^{2}\right] L
\end{aligned}
$$

and for each $x \in[1 / 2 \sqrt{3}, 1 / 2]$

$$
\left|\int_{0}^{1} f(t) d t-D(x)+\frac{B_{2}(x)}{2}\left[f^{\prime}(1)-f^{\prime}(0)\right]\right| \leq\left(-\frac{x^{3}}{6}+\frac{x^{2}}{8}-\frac{1}{192}\right) L .
$$

PROOF. Using (2.7) for each $x \in[0,1 / 2-1 / 2 \sqrt{3}]$ we get

$$
\begin{aligned}
\int_{0}^{1}\left|F_{3}^{x}(t)\right| d t & =2 \int_{0}^{1 / 2}\left|F_{3}^{x}(t)\right| d t=-2 \int_{0}^{1 / 2} F_{3}^{x}(t) d t=-2\left(-\left.\frac{1}{4} G_{4}^{x}(t)\right|_{0} ^{1 / 2}\right) \\
& =\frac{1}{2}\left[G_{4}^{x}\left(\frac{1}{2}\right)-\tilde{B}_{4}(x)\right]=\frac{1}{2} F_{4}^{x}\left(\frac{1}{2}\right),
\end{aligned}
$$

for each $x \in[1 / 2-1 / 2 \sqrt{3}, 1 / 4]$

$$
\begin{aligned}
\int_{0}^{1}\left|F_{3}^{x}(t)\right| d t & =-2 \int_{0}^{1 / 2} F_{3}^{x}(t) d t+4 \int_{0}^{\sqrt{-3 x^{2}+3 x-1 / 2}} F_{3}^{x}(t) d t \\
& =\frac{1}{2}\left[G_{4}^{x}(1 / 2)-2 G_{4}^{x}\left(\sqrt{-3 x^{2}+3 x-1 / 2}\right)+\tilde{B}_{4}(x)\right] \\
& =\frac{1}{2}\left[F_{4}^{x}(1 / 2)-2 F_{4}^{x}\left(\sqrt{-3 x^{2}+3 x-1 / 2}\right)\right],
\end{aligned}
$$

for each $x \in[1 / 4,1 / 2 \sqrt{3}]$

$$
\begin{aligned}
\int_{0}^{1}\left|F_{3}^{x}(t)\right| d t & =-2 \int_{0}^{1 / 2} F_{3}^{x}(t) d t+4 \int_{0}^{1-\sqrt{1-12 x^{2}} / 2} F_{3}^{x}(t) d t \\
& =\frac{1}{2}\left[G_{4}^{x}\left(\frac{1}{2}\right)-2 G_{4}^{x}\left(\frac{1-\sqrt{1-12 x^{2}}}{2}\right)+\tilde{B}_{4}(x)\right] \\
& =\frac{1}{2}\left[F_{4}^{x}\left(\frac{1}{2}\right)-2 F_{4}^{x}\left(\frac{1-\sqrt{1-12 x^{2}}}{2}\right)\right]
\end{aligned}
$$


and for each $x \in[1 / 2 \sqrt{3}, 1 / 2]$ we get

$$
\int_{0}^{1}\left|F_{3}^{x}(t)\right| d t=2 \int_{0}^{1 / 2} F_{3}^{x}(t) d t=-\frac{1}{2}\left[G_{4}^{x}(1 / 2)-\tilde{B}_{4}(x)\right]=-\frac{1}{2} F_{4}^{x}(1 / 2) .
$$

Therefore, applying (3.1) with $n=3$ and $p=\infty$, we get the above inequalities.

REMARK 6. Let $f:[0,1] \rightarrow \mathbb{R}$ be such that $f^{(n-1)}$ is an $L$-Lipschitzian function on $[0,1]$ for some $n \geq 3$. Then for each $x \in[0,1 / 2-1 / 2 \sqrt{3}) \cup(1 / 2 \sqrt{3}, 1 / 2]$, from Corollary 2.5 we get

$$
\begin{gathered}
K(2 k-1, \infty, x)=\frac{2}{(2 k) !}\left|B_{2 k}(1 / 2-x)-B_{2 k}(x)\right| \\
K *(2 k, \infty, x)=\frac{1}{(2 k) !}\left|B_{2 k}(x)\right| \text { and } K(2 k, \infty, x)=\frac{2}{(2 k) !}\left|B_{2 k}(x)\right| .
\end{gathered}
$$

If in the first inequality in Corollary 3.6 we put $k=2$ we get the same inequalities as in Corollary 3.6 when $x$ is from the intervals $[0,1 / 2-1 / 2 \sqrt{3})$ and $(1 / 2 \sqrt{3}, 1 / 2]$.

REMARK 7. If in Corollaries 3.3-3.6 and Remark 6 we choose $x=0,1 / 2,1 / 3$ we get inequalities related to the trapezoid (see $[3,6,12]$ ), the midpoint (see $[4,8,11]$ ) and the two-point Newton-Cotes formulae (see [17]), respectively. For $x=1 / 4$ in Corollaries 3.3-3.6 we get inequalities related to the two-point Maclaurin formulae (see [10]).

COROLLARY 3.7. Let $f:[0,1] \rightarrow \mathbb{R}$ be a given function. If $f$ is a continuous function of bounded variation on $[0,1]$, then for $x \in[0,1 / 2]$

$$
\left|\int_{0}^{1} f(t) d t-D(x)\right| \leq \frac{1+|4 x-1|}{4} V_{0}^{1}(f) .
$$

PROOF. From the explicit expressions (2.4) we have

$$
\max _{t \in[0,1]}\left|F_{1}^{x}(t)\right|=\max \{2 x,-2 x+1\}=\max \{A, B\},
$$

where $A=2 x, B=-2 x+1$. Also, $\max \{A, B\}=(A+B+|A-B|) / 2$, so using this formula and applying (3.1) with $n=1$ and $p=1$ we get the above inequality.

REMARK 8. The inequality (3.11) has been proved by Dragomir in [9].

COROLlaRY 3.8. Let $f:[0,1] \rightarrow \mathbb{R}$ be a given function. If $f^{\prime}$ is a continuous function of bounded variation on $[0,1]$, then for each $x \in[0,1 / 4]$

$$
\left|\int_{0}^{1} f(t) d t-D(x)\right| \leq \frac{4 x^{2}-4 x+1+\left|4 x^{2}+4 x-1\right|}{16} V_{0}^{1}\left(f^{\prime}\right)
$$


and for each $x \in[1 / 4,1 / 2]$

$$
\left|\int_{0}^{1} f(t) d t-D(x)\right| \leq \frac{x^{2}}{2} V_{0}^{1}\left(f^{\prime}\right) .
$$

Proof. From the explicit expressions (2.6) and for each $x \in[0,1 / 4]$ we have

$$
\max _{t \in[0,1]}\left|F_{2}^{x}(t)\right|=\max \left\{2 x^{2},-2 x+1 / 2\right\}
$$

and for each $x \in[1 / 4,1 / 2], \max _{t \in[0,1]}\left|F_{2}^{x}(t)\right|=2 x^{2}$. So using these two formulae and applying (3.1) with $n=2$ and $p=1$ we get inequalities (3.12) and (3.13).

COROLlaRY 3.9. Let $f:[0,1] \rightarrow \mathbb{R}$ be given. If $f^{\prime}$ is a continuous function of bounded variation on $[0,1]$, then for each $x \in[0,1 / 2]$

$$
\left|\int_{0}^{1} f(t) d t-D(x)+\frac{B_{2}(x)}{2}\left[f^{\prime}(1)-f^{\prime}(0)\right]\right| \leq\left(x^{2}-\frac{x}{2}+\frac{1}{12}\right) V_{0}^{1}\left(f^{\prime}\right) .
$$

Proof. Using (2.5) for each $x \in[0,1 / 2]$ we get

$$
\max _{i \in[0,1]}\left|G_{2}^{x}(t)\right|=\max \left\{\left|G_{2}(0)\right|,\left|G_{2}(x)\right|,\left|G_{2}(1 / 2)\right|\right\} .
$$

Therefore, applying (3.2) with $n=2$ and $p=1$, we get the above inequality.

REMARK 9. We mention here that comparing the best possible constant from Guessab and Schmeisser in [14] in the case $p=1$ and our constant, we conclude that inequality ( 3.5 ) is not generally best possible. Namely, our constant for boundary conditions $f^{\prime}(1)=f^{\prime}(0), n=2$ and $x=0$ is $1 / 12$, while they have $1 / 16$.

COROLLARY 3.10. Let $f:[0,1] \rightarrow \mathbb{R}$ be given. If $f^{\prime \prime}$ is a continuous function of bounded variation on $[0,1]$, then for each $x \in[0,1 / 4]$

$$
\left|\int_{0}^{1} f(t) d t-D(x)+\frac{B_{2}(x)}{2}\left[f^{\prime}(1)-f^{\prime}(0)\right]\right| \leq \frac{1}{72 \sqrt{3}}\left(1-12 x^{2}\right)^{3 / 2} V_{0}^{1}\left(f^{\prime \prime}\right)
$$

and for each $x \in[1 / 4,1 / 2]$

$\left|\int_{0}^{1} f(t) d t-D(x)+\frac{B_{2}(x)}{2}\left[f^{\prime}(1)-f^{\prime}(0)\right]\right| \leq \frac{1}{3}\left(-x^{2}+x-\frac{1}{6}\right)^{3 / 2} V_{0}^{\prime}\left(f^{\prime \prime}\right)$.

PROOF. Using (2.7) for each $x \in[0,1 / 2-1 / 2 \sqrt{3}]$ we get

$$
\max _{t \in[0,1]}\left|F_{3}^{x}(t)\right|=\left|F_{3}\left(\frac{1}{2}-\frac{\sqrt{1-12 x^{2}}}{2 \sqrt{3}}\right)\right| .
$$


for each $x \in[1 / 2-1 / 2 \sqrt{3}, 1 / 4]$

$$
\max _{t \in[0,1]}\left|F_{3}^{x}(t)\right|=\max \left\{\left|F_{3}\left(\frac{1}{2}-\frac{\sqrt{1-12 x^{2}}}{2 \sqrt{3}}\right)\right|,\left|F_{3}\left(\sqrt{-x^{2}+x-\frac{1}{6}}\right)\right|\right\},
$$

for each $x \in[1 / 4,1 / 2 \sqrt{3}]$

$$
\max _{t \in[0,1]}\left|F_{3}^{x}(t)\right|=\max \left\{\left|F_{3}\left(\frac{1}{2}-\frac{\sqrt{1-12 x^{2}}}{2 \sqrt{3}}\right)\right|,\left|F_{3}\left(\sqrt{-x^{2}+x-\frac{1}{6}}\right)\right|\right\}
$$

and for each $x \in[1 / 2 \sqrt{3}, 1 / 2]$ we get

$$
\max _{i \in[0,1]}\left|F_{3}^{x}(t)\right|=\left|F_{3}\left(\sqrt{-x^{2}+x-1 / 6}\right)\right| .
$$

Therefore, applying (3.1) with $n=3$ and $p=1$, we get the above inequalities.

REMARK 10. Let $f:[0,1] \rightarrow \mathbb{R}$ be such that $f^{(n-1)}$ is a continuous function of bounded variation on $[0,1]$ for some $n \geq 3$. Then for each $x \in[0,1 / 2-1 / 2 \sqrt{3}) \cup$ $(1 / 2 \sqrt{3}, 1 / 2]$, from Corollary 2.4 we get

$$
\begin{aligned}
K(2 k-1,1, x) & =\frac{1}{2(2 k-1) !} \max _{t \in[0,1]}\left|F_{2 k-1}^{x}(t)\right|, \\
K^{*}(2 k, 1, x) & =\frac{1}{(2 k) !}\left|B_{2 k}(1 / 2-x)-B_{2 k}(x)\right| \text { and } \\
K(2 k-1,1, x) & =\frac{1}{(2 k) !} \max \left\{\left|B_{2 k}(x)\right|,\left|B_{2 k}(1 / 2-x)\right|\right\} .
\end{aligned}
$$

If in the first inequality in Corollary 3.10 we put $k=2$ we get the same inequalities as in Corollary 3.10 when $x$ is from the intervals $[0,1 / 2-1 / 2 \sqrt{3})$ and $(1 / 2 \sqrt{3}, 1 / 2]$.

REMARK 11. If in Corollaries 3.7-3.10 and Remark 10 we choose $x=0,1 / 2,1 / 3$ we get inequalities related to the trapezoid (see $[3,6,12])$, the midpoint (see $[4,8,11])$ and the two-point Newton-Cotes formulae (see [17]), respectively. For $x=1 / 4$ in Corollaries 3.7-3.10 we get inequalities related to the two-point Maclaurin formulae (see [9]).

Now, we calculate the optimal constant for $p=2$.

COROLLARY 3.11. Let $\left|f^{(n)}\right|^{2}:[0,1] \rightarrow \mathbb{B}$ be a $R$-integrable function for some $n \geq 1$. Then for each $x \in[0,1 / 2]$, we have

$$
\begin{aligned}
& \left|\int_{0}^{1} f(t) d t-D(x)+\tilde{T}_{n-1}(x)\right| \\
& \quad \leq \frac{1}{2}\left[\frac{2(-1)^{n-1}}{(2 n) !}\left[B_{2 n}+B_{2 n}(1-2 x)\right]+\frac{4}{(n !)^{2}} B_{n}^{2}(x)\right]^{1 / 2}\left\|f^{(n)}\right\|_{2}
\end{aligned}
$$


and

$\left|\int_{0}^{1} f(t) d t-D(x)+\tilde{T}_{n}(x)\right| \leq \frac{1}{2}\left[\frac{2(-1)^{n-1}}{(2 n) !}\left[B_{2 n}+B_{2 n}(1-2 x)\right]\right]^{1 / 2}\left\|f^{(n)}\right\|_{2}$.

PROOF. Using integration by parts and also using [5, Lemma 1] we have

$$
\begin{aligned}
\int_{0}^{1} G_{n}^{x 2}(t) d t & =(-1)^{n-1} \frac{n(n-1) \cdots 2}{(n+1)(n+2) \cdots(2 n-1)}\left[\frac{1}{2 n} \int_{0}^{1} G_{2 n}^{x}(t) d G_{1}^{x}(t)\right] \\
& =(-1)^{n-1} \frac{(n !)^{2}}{(2 n) !}\left[-2 \int_{0}^{1} G_{2 n}^{x}(t) d t+G_{2 n}^{x}(x)+G_{2 n}^{x}(1-x)\right] \\
& =(-1)^{n-1} \frac{2(n !)^{2}}{(2 n) !}\left[B_{2 n}+B_{2 n}(1-2 x)\right] .
\end{aligned}
$$

Now,

$$
\begin{aligned}
\int_{0}^{1} F_{n}^{x^{2}}(t) d t & =\int_{0}^{1}\left[G_{n}^{x}(t)-\tilde{B}_{n}(x)\right]^{2} d t \\
& =\int_{0}^{1}\left[G_{n}^{x^{2}}(t)-2 G_{n}^{x}(t) \tilde{B}_{n}(x)+\tilde{B}_{n}^{2}(x)\right] d t \\
& =\int_{0}^{1} G_{n}^{x 2}(t) d t+\tilde{B}_{n}^{2}(x) \\
& =(-1)^{n-1} \frac{2(n !)^{2}}{(2 n) !}\left[B_{2 n}+B_{2 n}(1-2 x)\right]+4 B_{n}^{2}(x)
\end{aligned}
$$

REMARK 12. For $n=2$ we have the boundary conditions $f^{\prime}(1)=f^{\prime}(0)$. For $x=0$ our constant from Theorem 3.2 is $1 /(12 \sqrt{3})$. Guessab and Schmeisser in [14] also have $1 /(12 \sqrt{3})$ which confirms the sharpness of our inequality in this case.

Finally, we give the values of the optimal constant for $n=1$ and arbitrary $p$ from Theorem 3.1.

REMARK 13. Note that $K^{*}(1, p, x)=K(1, p, x)$, for $1<p \leq \infty$, since $G_{1}^{x}(t)=$ $F_{1}^{x}(t)$. Also, for $1<p \leq \infty$ we can easily calculate $K(1, p, x)$. We get

$$
K(1, p, x)=\frac{1}{2}\left[\frac{(2 x)^{q+1}+(1-2 x)^{q+1}}{q+1}\right]^{1 / q}, \quad 1<p \leq \infty
$$

REMARK 14. Equality (3.14) has been proved by Dragomir on the interval $[a, b]$ in [10].

REMARK 15. If in Remark 13 we choose $x=0,1 / 2,1 / 3,1 / 4$ we get inequalities related to the trapezoid (see [6]), the midpoint (see [8]), the two-point Newton-Cotes (see [17]) and the two-point Maclaurin formulae (see [10]), respectively. 
In the following theorem we use (2.2) and a technical result from the recent paper [16] to obtain a Grüss-type inequality related to the general Euler two-point formula (see [16]).

THEOREM 3.12. Suppose that $f:[0,1] \rightarrow \mathbb{R}$ is such that $f^{(n)}$ exists and is integrable on $[0,1]$, for some $n \geq 1$. Assume that $m_{n} \leq f^{(n)}(t) \leq M_{n}, 0 \leq t \leq 1$, for some constants $m_{n}$ and $M_{n}$. Then for $x \in[0,1 / 2]$

$$
\left|\int_{0}^{1} f(t) d t-D(x)+\tilde{T}_{n}(x)\right| \leq C_{n}\left(M_{n}-m_{n}\right),
$$

where $C_{n}=(1 / 4(n !)) \int_{0}^{1}\left|G_{n}^{x}(t)\right| d t$.

REMARK 16. If in Theorem 3.12 we choose $x=0,1 / 2,1 / 3$ we get inequalities related to the trapezoid, the midpoint and the two-point Newton-Cotes formulae (see [16]). For $x=1 / 4$ we get inequalities related to the two-point Maclaurin formulae.

Our final results are connected with the series expansion of a function in Bernoulli polynomials.

THEOREM 3.13. If $f:[0,1] \rightarrow \mathbb{B}$ is such that $f^{(2 k)}$ is a continuous function on $[0,1]$, then for some $k \geq 2$ there exists a point $\eta \in[0,1]$ such that

and

$$
\tilde{R}_{2 k}^{2}(f)=\frac{B_{2 k}(x)}{[(2 k) !]} f^{(2 k)}(\eta) \quad \text { for } x \in[0,1 / 2-1 / 2 \sqrt{3})
$$

$$
\tilde{R}_{2 k}^{2}(f)=-\frac{B_{2 k}(x)}{[(2 k) !]} f^{(2 k)}(\eta) \text { for } x \in(1 / 2 \sqrt{3}, 1 / 2] .
$$

Proof. We can rewrite $\tilde{R}_{2 k}^{2}(f)$ for $x \in[0,1 / 2-1 / 2 \sqrt{3})$ as

where

$$
\tilde{R}_{2 k}^{2}(f)=(-1)^{k} \frac{J_{k}}{2[(2 k) !]},
$$

$$
J_{k}=\int_{0}^{1}(-1)^{k} F_{2 k}^{x}(s) f^{(2 k)}(s) d s
$$

From Corollary 2.4 it follows that $(-1)^{k} F_{2 k}^{x}(s) \geq 0,0 \leq s \leq 1$ and the claim follows from the mean value theorem for integrals and Corollary 2.5. The proof on the interval $(1 / 2 \sqrt{3}, 1 / 2]$ is similar. 
REMARK 17. For $k=2$ formulae (3.16) and (3.17) reduce to

$$
\tilde{R}_{4}^{2}(f)=\frac{B_{4}(x)}{24} f^{(4)}(\eta) \quad \text { and } \quad \tilde{R}_{4}^{2}(f)=-\frac{B_{4}(x)}{24} f^{(4)}(\eta),
$$

respectively, which are formulae proved for $x=0$ in [6], for $x=1 / 2$ in [8] and for $x=1 / 3$ in [17].

COROLlARY 3.14. Let $f \in C^{\infty}[0,1]$ and $\lambda \in \mathbb{B}$ be such that $0<\lambda<2 \pi$ and $\left|f^{(2 k)}(t)\right| \leq \lambda^{2 k}$ for $t \in[0,1]$ and $k \geq k_{0}$ for some $k_{0} \geq 2$. Then for $x \in$ $[0,1 / 2-1 / 2 \sqrt{3}) \cup(1 / 2 \sqrt{3}, 1 / 2]$ we have

$$
\int_{0}^{1} f(t) d t=D(x)-\frac{1}{2} \sum_{j=1}^{\infty} \frac{B_{2 j}(x)}{(2 j) !}\left[f^{(2 j-1)}(1)-f^{(2 j-1)}(0)\right] .
$$

Proof. From Theorem 3.13 when $k \geq k_{0}$ we have that

$$
\left|\tilde{R}_{2 k}^{2}(f)\right| \leq \frac{\left|B_{2 k}(x)\right|}{(2 k) !} \lambda^{2 k} \leq \frac{\left|B_{2 k}\right|}{(2 k) !} \lambda^{2 k} \approx \frac{1}{(2 k) !} 2 \frac{(2 k) !}{(2 \pi)^{2 k}} \lambda^{2 k}=2\left(\frac{\lambda}{2 \pi}\right)^{2 k},
$$

so (3.18) follows.

\section{References}

[1] M. Abramowitz and I. A. Stegun (eds.), Handbook of mathematical functions with formulae, graphs and mathematical tables, Appl. Math. Series 55 (National Bureau of Standards, Washington, 1965).

[2] I. S. Berezin and N. P. Zhidkov, Computing methods, Vol. I (Pergamon Press, Oxford, 1965).

[3] P. Cerone and S. S. Dragomir, "Trapezoidal type rules from an inequalities point of view", RGMIA 2 (1999) Article 8.

[4] P. C. Cerone and S. S. Dragomir, "Midpoint-type rules from an inequalities point of view", in Handbook of analytic-computational methods in applied mathematics, (Chapman \& Hall/CRC, Boca Raton, FL, 2000) 135-200.

[5] Lj. Dedic, M. Matič and J. Pečric, "On generalizations of Ostrowski inequality via some Euler-type identities", Math. Inequal. Appl. 3 (2000) 337-353.

[6] Lj. Dedić, M. Matić and J. Pečarić, "On Euler trapezoid formulae", Appl. Math. Comput. 123 (2001) 37-62.

[7] Lj. Dedic, M. Matić and J. Pečarić, "Some inequalities of Euler-Grüss type", Comput. Math. Appl. 41 (2001) 843-856.

[8] Lj. Dedić, M. Matić and J. Pečarić, “On Euler midpoint formulae”, ANZIAM J. 46 (2005) 417-438.

[9] S. S. Dragomir, "A companion of Ostrowski's inequality for functions of bounded variation and applications", RGMIA 5 (2002) supplement.

[10] S. S. Dragomir, "Some companions of Ostrowski's inequality for absolutely continuous functions and applications", RGMIA 5 (2002) supplement. 
[11] S. S. Dragomir, P. Cerone and A. Sofo, "Some remarks on the midpoint rule in numerical integration", Studia Math. Babes-Bolyai Univ. 45 (2000) 63-73.

[12] S. S. Dragomir, P. Cerone and A. Sofo, "Some remarks on the trapezoid rule in numerical integration", Indian J. Pure Appl. Math. 31 (2000) 475-494.

[13] A. M. Fink, "Bounds on the deviation of a function from its averages", Czechoslovak Math. J. 42 (1992) 289-310.

[14] A. Guessab and G. Schmeisser, "Sharp integral inequalities of the Hermite-Hadamard type", J. Approx. Theory 115 (2002) 260-288.

[15] V. I. Krylov, Approximate calculation of integrals (Macmillan, New York-London, 1962).

[16] M. Matic, "Improvement of some inequalities of Euler-Grüss type", Comput. Math. Appl. 46 (2003) 1325-1336.

[17] M. Matic, C. E. M. Pearce and J. Pečaric, "Two-point formulae of Euler type", ANZIAM J. 44 (2002) 221-245. 